\section{FORECAST: A general purpose computer aid to judgmental forecasting}

\section{GEORGE WRIGHT, PETER AYTON, and PETER WHALLEY \\ City of London Polytechnic, London, England}

The elicitation of subjective probability as a means of gaining access to an individual's internal state of uncertainty is a procedure commonly used in decision-aiding techniques (see Wright, 1984). By this means expert judgment may be tapped. Experimental psychologists have also used this method for exploring the characteristics of human judgment. Judgments expressed as subjective probabilities must conform to the probability laws. However, Tversky and Kahneman (1983) and Wright and Whalley (1983) have shown that actual probabilistic judgments of future event likelihood frequently contradict axioms derived from probability theory. In the former study, this contradiction was shown even in judgments given by professional statisticians. The program described here takes advantage of some axioms of probability theory to enable the user to make coherent judgments of the probability of future events. As incoherent (i.e., axiom-breaking) forecast probabilities cannot be accurate, the program interacts with the user to resolve inconsistencies in a manner that meets with the user's approval.

The program FORECAST aids forecasting of (1) a time period or date when a specified event may happen (e.g., the United States dollar reaches parity with the pound sterling next month); (2) the possible outcomes of an event, expressed in numerical terms as outcomes on a single continuous scale (e.g., company profits); and (3) possible outcomes of an event, expressed as discrete or discontinuous outcomes (e.g., the next prime minister of Great Britain). The user selects from a menu the type of forecast he or she wishes to make, and the appropriate procedure is initiated.

Time-Period Forecasting. For time-period forecasting, the program elicits from the user the beginning and end of the time period of interest (time period B). Next the program elicits a point-probability estimate and an odds estimate for the event happening at least once in the forecast period (e.g., the pound sterling falling to a value of less than $\$ 1.00$ ). These formally equivalent estimates are compared by the program, and inconsistencies are reported to the user for resolution to give $P(B)$. Thus, the user is made aware of error at the level of the mode of elicitation. Next, the program attempts to extend the time period of the forecast by a subsequent time interval consisting of the same number of days as the main time period of

This work was supported by Grant $\mathrm{C0023207}$ from the ESRC. The authors' mailing address is: Psychology Department, City of London Polytechnic, Old Castle Street, London, England E1 7NT. interest (time period $\mathrm{C}$ ). The program then elicits $\mathrm{P}(\mathrm{C})$. By consulting a calendar, the program investigates the possibility of the event occurring at least once in a similarlength time period prior to the main time period of interest, providing this time period does not extend into the past (time period $A$ ). The program elicits $P(A)$ and then divides in half the main time period of interest, period $B$, and elicits two further forecasts, $P(D)$ and $P(E)$.

At the next stage, the program investigates whether the perceived likelihood of the event occurring in each of the subsequent time periods is independent or dependent on the event's having occurred in the prior time period. If the assessments are seen as conditional, the program elicits the conditional probabilities: $\mathbf{P}(\mathrm{B} / \mathrm{A}), \mathrm{P}(\mathrm{B} / \mathrm{A}), \mathrm{P}(\mathrm{C} / \mathrm{B})$, $P(C / \bar{B}), P(E / D)$, and $P(E / \bar{D})$.

The program subsequently elicits a forecast of the event's happening at least once in each of the paired time periods [i.e., $P(A$ and $B), P(B$ and $C)$, and $P(D$ and $E)$ ]. Next the program elicits forecasts of the event's happening in one or both of the combined time periods $B$ and $C$ or in combined periods $A$ and $B$ [i.e., $P(B$ and/or $C)$ and $\mathrm{P}(\mathrm{A}$ and/or $\mathrm{B})]$. Finally the program elicits forecasts of the event's happening at least once in one (but not both) of two time intervals [i.e., $\mathrm{P}(\mathrm{A}$ or $\mathrm{B}), \mathrm{P}(\mathrm{B}$ or $\mathrm{C})$ and $\mathrm{P}(\mathrm{D}$ or $\mathrm{E})]$.

Figure 1 gives a summary of the program's output. The first probability of each pair is an intuitive holistic forecast; the second probability expression is the "equivalent" normative probability forecast recomposed from the user's input to the program.

\section{$\mathbf{P}(\mathrm{A}$ and $\mathrm{B}) \quad \mathbf{P}(\mathrm{A}) \mathbf{P}(\mathrm{B} / \mathrm{A})$}

$P(B$ and $C) \quad P(B) P(C / B)$

$P(D$ and $E) \quad P(D) P(E / D)$

$\mathbf{P}(\mathbf{A}$ or $\mathbf{B}) \quad \mathbf{P}(\mathbf{A}) \mathrm{P}[1-(\mathrm{B} / \mathrm{A})]+\mathbf{P}(1-\mathbf{A}) \mathbf{P}(\mathbf{B} / \overline{\mathbf{A}})$

$\mathrm{P}(\mathrm{B}$ or $\mathrm{C}) \quad \mathrm{P}(\mathrm{B}) \mathrm{P}[1-(\mathrm{C} / \mathrm{B})]+\mathrm{P}(1-\mathrm{B}) \mathrm{P}(\mathrm{C} / \overline{\mathrm{B}})$

$P(D$ or $E) \quad P(D) P[1-(E / D)]+P(1-D) P(E / \bar{D})$

$\mathbf{P}(\mathrm{B}$ and/or $\mathrm{C}) \quad \mathrm{P}(\mathrm{B}) \mathrm{P}(\mathrm{C} / \mathrm{B})+\mathrm{P}(\mathrm{B}) \mathrm{P}[1-(\mathrm{C} / \mathrm{B})]+\mathrm{P}(1-\mathrm{B}) \mathrm{P}(\mathrm{C} / \overline{\mathrm{B}})$

$\mathbf{P}(\mathbf{A}$ and/or $\mathbf{B}) \quad \mathbf{P}(\mathbf{A}) \mathbf{P}(\mathbf{B} / \mathbf{A})+\mathbf{P}(\mathbf{A}) \mathbf{P}[1-(\mathbf{B} / \mathbf{A}]+\mathbf{P}(1-\mathbf{A}) \mathbf{P}(\mathbf{B} / \overline{\mathbf{A}})$

$P(D) P(E / D)+P(D) P[1-(E / D)]+P(1-D) P(E / \bar{D})$

Figure 1. Summary of program output for time-period forecasting. 
Continuous-Variable Forecasting. After the main menu selection has been made, the program elicits the minimum and maximum values of the range of outcomes. The program also verifies that the user's minima and maxima are as defined by asking for probability estimates for outcomes outside of this interval. Next, the program interactively subdivides this range into an acceptable number of divisions and proceeds to elicit forecasts for the subranges. The program reports assessed probabilities to the user and, if the assessments are not additive, presents two normalizations, N1 and N2. N1 equally allocates the computed amount of nonadditivity among the subrange forecasts. N2 shares any nonadditivity proportionally according to the value of the original subrange forecasts. The program enquires whether either of the normalizations is acceptable or whether the user wishes to change his own forecasts to achieve additivity. Finally the program reports cumulative forecasts to the user.

Discrete-Variable Forecasting. After the user selects discrete-variable forecasting from the main menu, the program asks for an event name and a list of all possible outcomes. The program adds a "catchall" outcome to the user's outcome listing and elicits assessment for the likelihood of the outcomes, initially using both point probabilities and odds as response modes. If any probability above 0.01 is placed in the "catchall" outcome, the program prompts for further decomposition into specified possible outcomes. As with the continuous-variable forecasting option, the program next presents assessed probabilities and, if the assessments are not additive, presents normalizations $\mathrm{N} 1$ and $\mathrm{N} 2$ to the user for evaluation.

\section{Discussion}

Figure 2 shows the essential logic of the procedures underlying FORECAST. The program decomposes holistic forecasts and recomposes these by means of the nor-

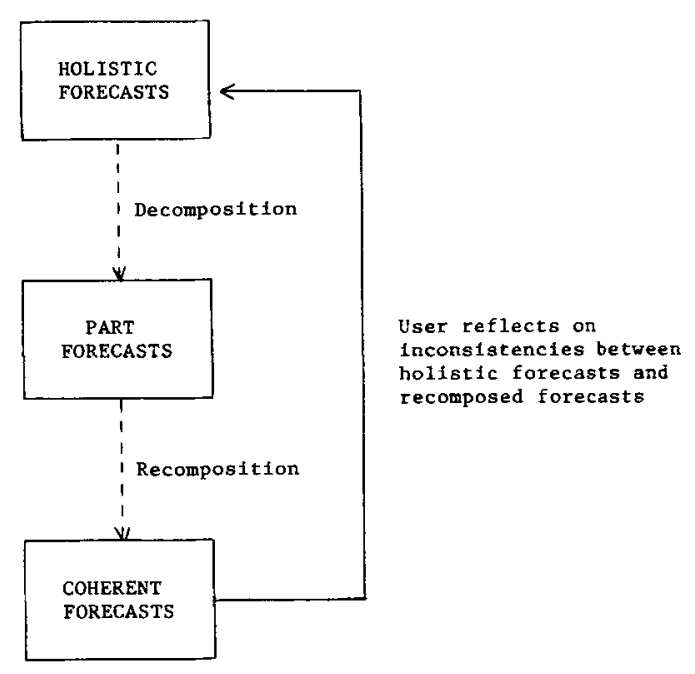

Figure 2. The essential logic of the FORECAST program. mative probability laws. Recomposed coherent forecasts are then presented to the forecaster together with his or her holistic forecasts. If there are discrepancies, the forecaster is given the opportunity to reflect on these discrepancies.

On reflection, the user may decide to accept the recomposed forecasts provided by the program, retain the original holistic forecasts, adjust the holistic forecasts in the light of presented recompositions, or start the program again. Except where normalizations ${ }^{1}$ are offered, the program offers no explicit guidance for resolving incoherence. It is possible to derive a rationale, similar to that given for subjective expected utility (SEU) theory, for the hypothesis that the coherent recomposed forecasts should be better than the holistic forecasts as they are based on simpler mental judgments. However, this hypothesis is by no means compelling and is in any case unlikely to apply for all types of forecast. A full discussion of the psychological context of the program is given in Wright, Ayton, and Whalley (in press). We consider it valuable for the forecasters to attempt to amend the judgments for themselves; in this way the forecasters' internal model of the events, even if revised by the process, is not compromised by the forecasts it should be accountable for. We are currently investigating the effects on calibration of interaction with the program. Calibration is one measure of the validity of subjective probability assessments (see Lichtenstein, Fischhoff, and Phillips, 1982, and Wright, 1982). For perfect calibration, assessed probability should equal percentage correct over a number of assessments of equal probability. For example, if a probability of occurrence of 0.7 is assigned to each of ten events, then seven of those events should occur. Similarly, all events assessed as being certain to occur (1.0 probability assessments) should occur. Coherence can be thought of as one measure of the reliability of probability forecasts. Logically, incoherent or unreliable forecasts cannot be better calibrated, or more valid, than coherent forecasts derived from the same knowledge. Therefore, we would expect people whose probability forecasts are incoherent to show poor forecasting performance.

One of the major uses of our program may be when sensitivity analyses, considered at an early stage in the elicitation of probabilities for a decision tree, indicate that small changes in "critical" probabilities have a major impact on which act is favored by subjective expected-utility theory (Raiffa, 1968). FORECAST is ideally suited to an in-depth examination of the coherence of subjective predictions and also has application in cross-impact analysis (Dalkey, 1972) and fault-tree analysis (Fischhoff, Slovic, \& Lichtenstein, 1978).

\section{Program Availability}

The program is documented and currently running on an Apple IIe microcomputer. It is written in BASIC, and conversion for an IBM personal computer is straightforward. A listing of the program is available from the authors at cost. 


\section{REFERENCES}

DalKeY, N. (1972). An elementary cross-impact model. Technological Forecasting \& Social Change, 3, 341-351.

Fischnoff, B., Slovic, P., \& Lichtenstein, S. (1978). Fault trees: Sensitivity of estimated failure probabilities to problem representation. Journal of Experimental Psychology: Human Perception \& Performance, 4, 330-344.

Lichtenstein, S., Fischhoff, B., \& Phillips, L. D. (1982). Calibration of probabilities: The state of the art to 1980. In D. Kahneman, P. Slovic, \& A. Tversky (Eds.), Judgment under uncertainty: Heuristics and biases. New York: Cambridge University Press.

Lindley, D. V., TVERSKY, A., \& Brown, R. V. (1979). On the reconciliation of probability assessments. Joumal of the Royal Statistical Society, 142, 146-180.

RAIFFA, H. (1968). Decision analysis-Introductory lectures on choices under uncertainty. Reading, MA: Addison-Wesley.

Tversky, A., \& Kahneman, D. (1983). Extensional versus intuitive reasoning: The conjunction fallacy in probability judgment. Psychological Review, 90, 293-315.

WRIGHT, G. (1982). Changes in the realism and distribution of proba- bility assessments as a function of question type. Acta Psychologica, 52, 165-174.

WRIGHT, G. (1984). Behavioral decision theory. London: Penguin.

Wright, G., Ayton, P., \& Whalley, P. (in press). A general purpose computer aid to judgmental forecasting: Rationale and Procedures. Decision Support Systems. Beverly Hills: Sage.

Wright, G., \& WALLEY, P. (1983). The supra-additivity of subjective probability. In B. P. Stigum and F. Wenstop (Eds.), Foundations of utility and risk theory with application. Dordrecht: Reidel.

\section{NOTE}

1. Obviously, there are many ways of altering a set of numbers so that they combine to some value [see Lindley, Tversky, \& Brown (1979) for a discussion of the problems in amending probabilities]. As the normalizations offered are somewhat arbitrary, they offer no ideal solution to incoherence, but they do illustrate possibilities which the forecaster may reject but, nonetheless, find useful to contemplate.

(Revision accepted for publication July 11, 1985). 